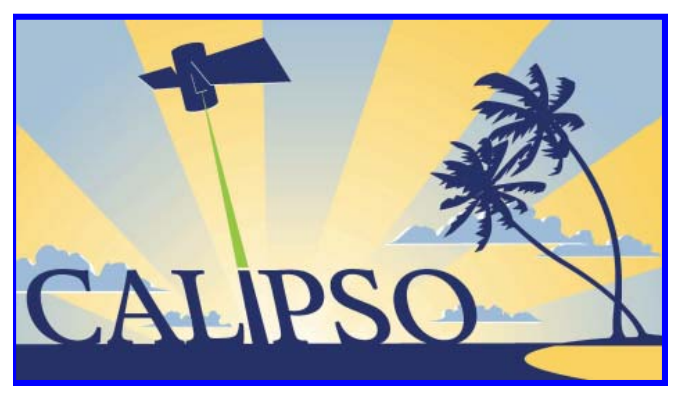

\title{
Aerosol properties from combined oxygen A band radiances and lidar
}

\author{
Dave Winker ${ }^{1}$, Peng-Wang Zhai ${ }^{2}$ and \\ Yongxiang $\mathrm{Hu}^{1}$ \\ ${ }^{1}$ NASA Langley Research Center, Hampton, VA \\ ${ }^{2} \mathrm{UMBC}$, Baltimore, MD
}




\section{MOTIVATION}

- Much of the uncertainty in estimating global direct aerosol radiative forcing comes from uncertainties in aerosol absorption

- Current satellite retrieval capabilities are very limited

- Multi-angle polarimetric methods have received a lot of attention

- High spectral resolution $\mathrm{O}_{2}$ A-band spectra offer another possibility






\section{High spectral resolution required}

Column $\mathrm{O}_{2}$ OD from line-by-line calculations

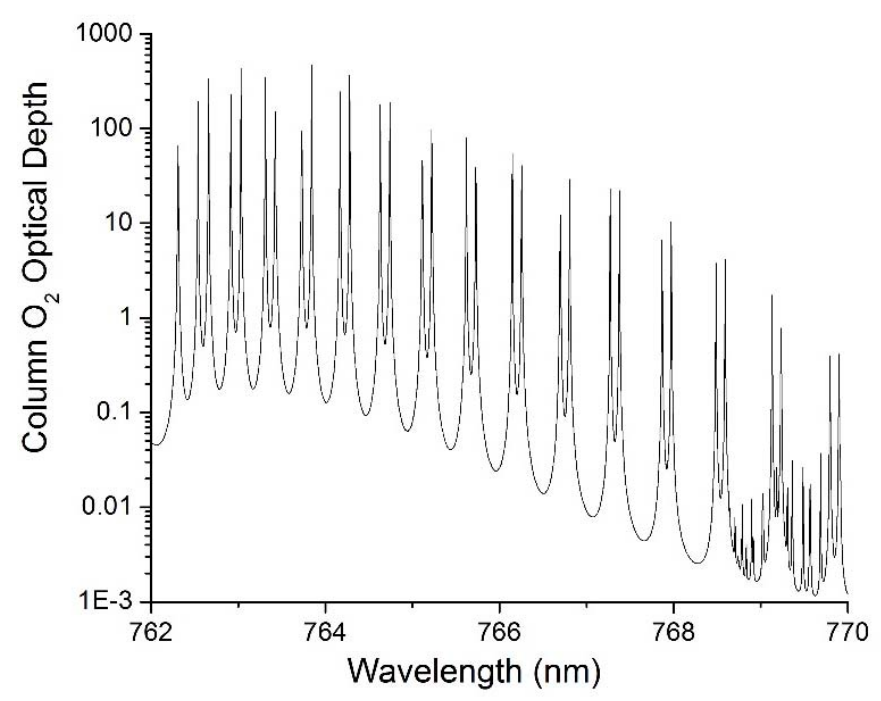

Atmospheric transmission at the resolution of OCO-2 spectrometer

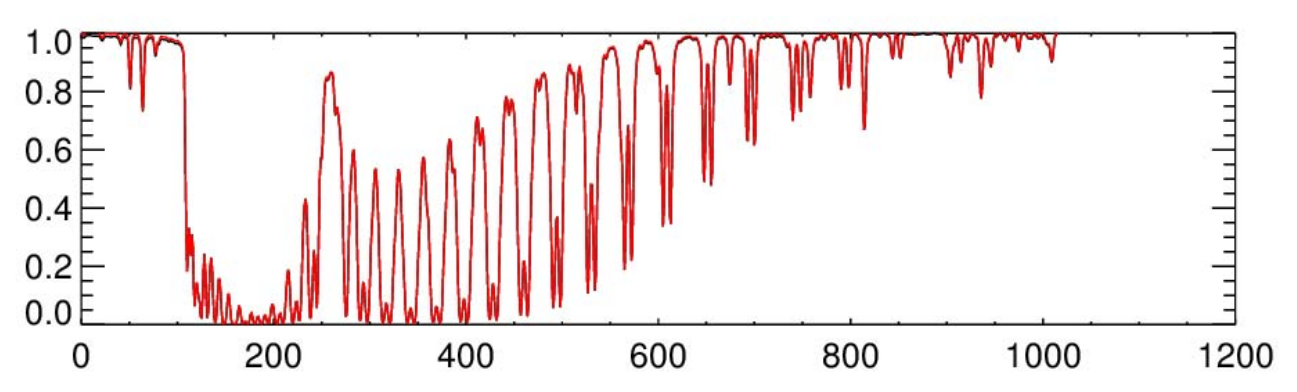

When the maximum column oxygen optical depth reaches $\sim 5$, transmission to the surface is very low and A-band measurements can be used to separate surface and atmospheric scattering. 


\section{Both CALIPSO and CloudSat originally included A-band spectrometers for cloud and aerosol retrievals}

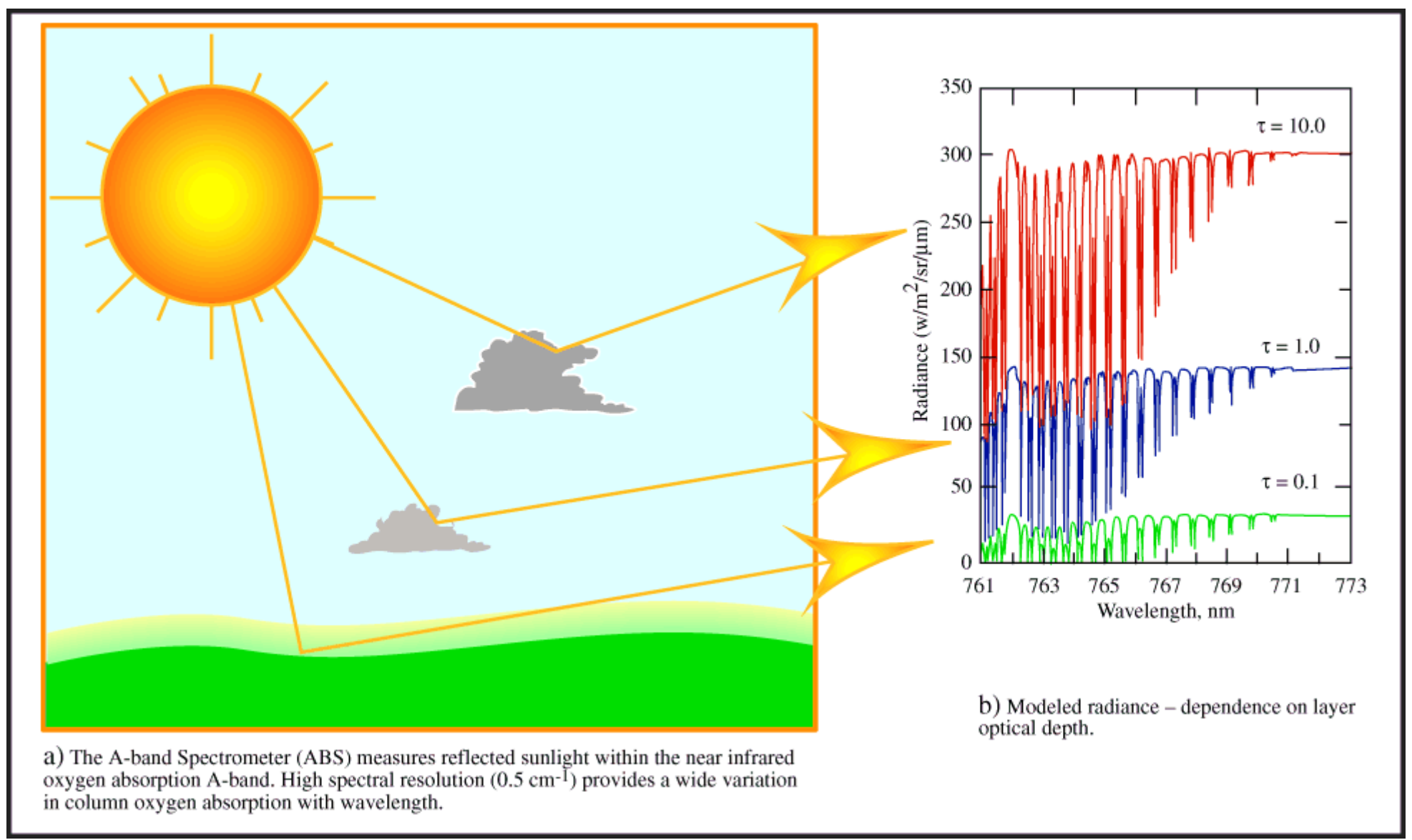




\section{A short history of A-band}

- Unique potential of reflected $\mathrm{O}_{2} \mathrm{~A}$-band radiances (for cloud height retrievals) was recognized in the early 1960's

- First satellite measurements: 1965, Gemini V

- Many instruments with A-band channels have flown since, but mostly with low spectral resolution

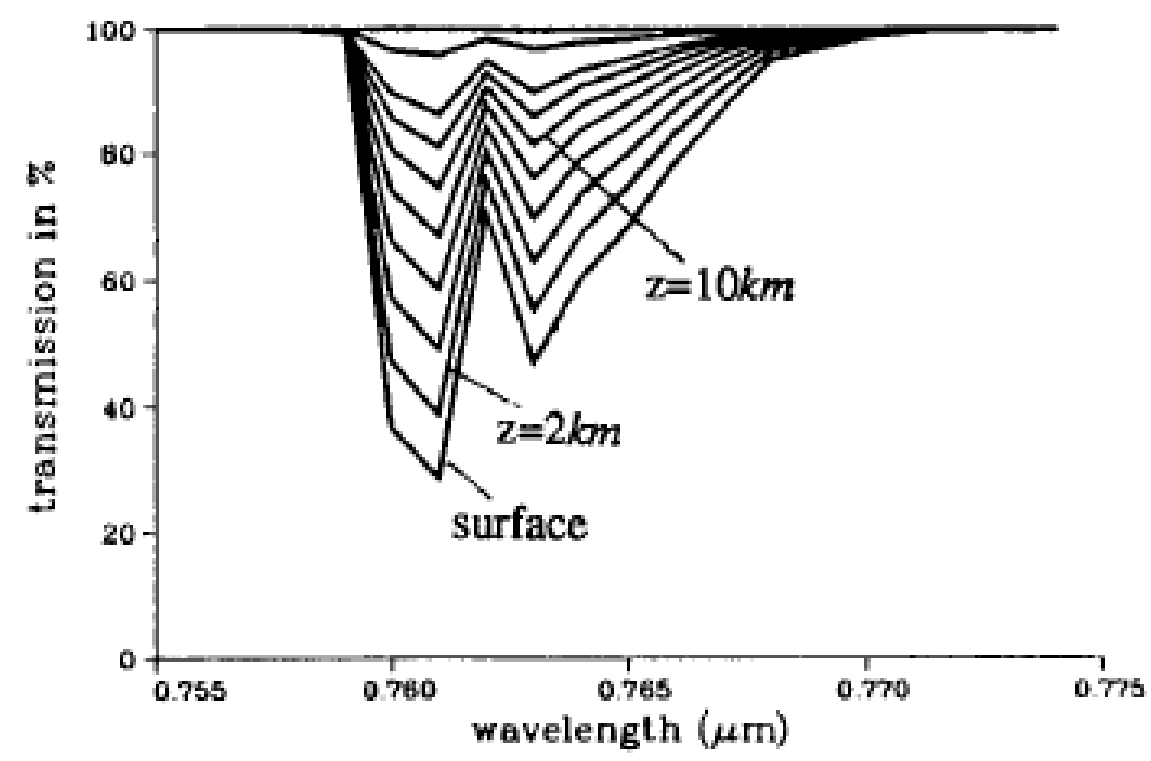

A-band spectrum at 1-nm resolution

(J. Fischer and H. Grassl, 1991) 


\section{High resolution A-band spectra}

- O'Brien and Mitchell (1992) were the first to realize the advantages of high spectral resolution A-band measurements

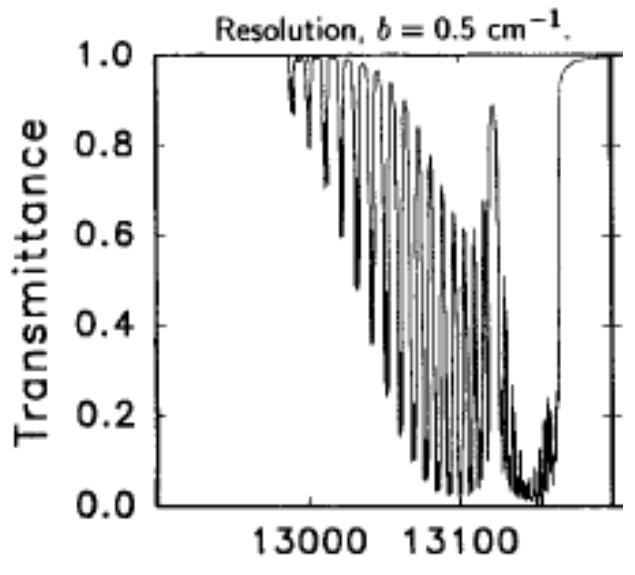

Resolution, $b=16.0 \mathrm{~cm}^{-1}$.

- With resolution $<0.5 \mathrm{~cm}^{-1}$, maximum $\mathrm{O}_{2}$ optical depth exceeds 5

- Combining weak and strong channels allows separating surface and atmospheric scattering.


(Stephens and Heidinger, JAS, 2000) 


\section{Retrieval Sensitivities}

The A band offers 2 critical advantages:

A-band weighting functions

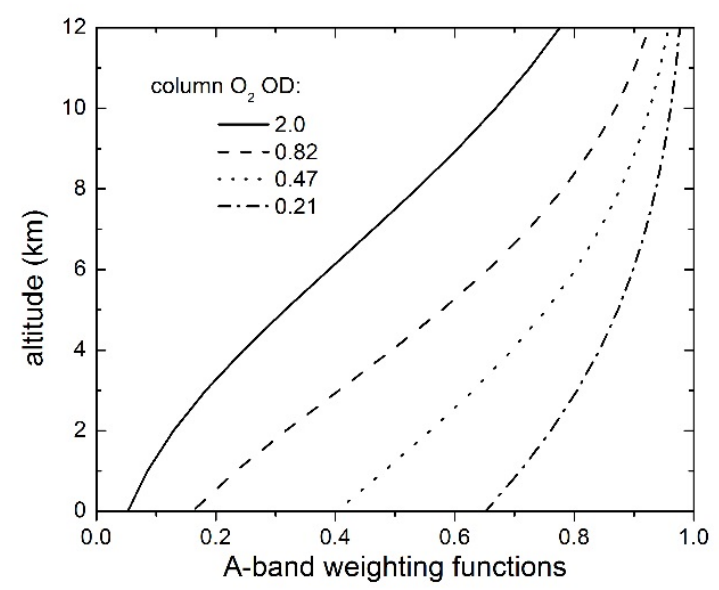

Sensitivity to surface and aerosol scattering

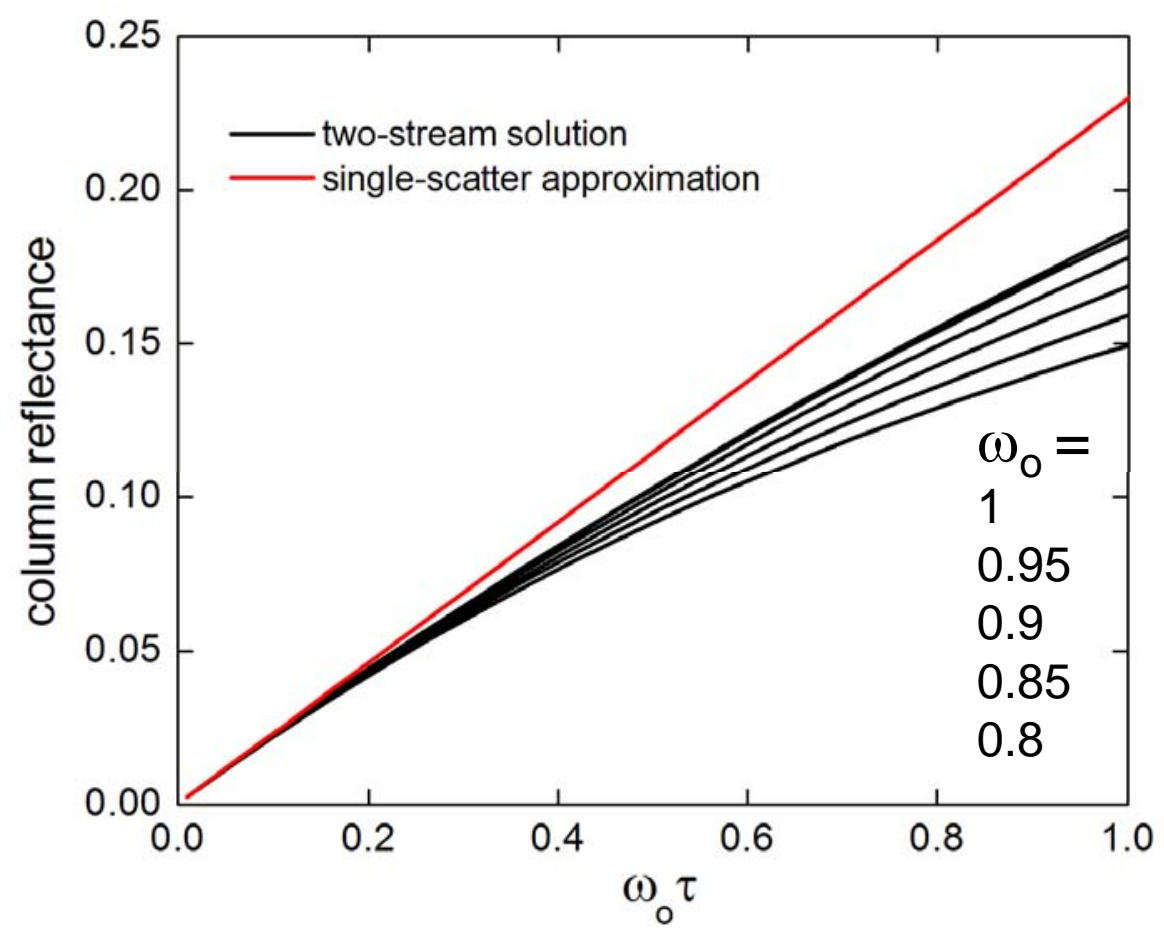

As OD increases, multiple scattering breaks the degeneracy between $\omega_{0}$ and $\tau$ 
Impacts of differences in aerosol scattering are small relative to impacts from aerosol absorption
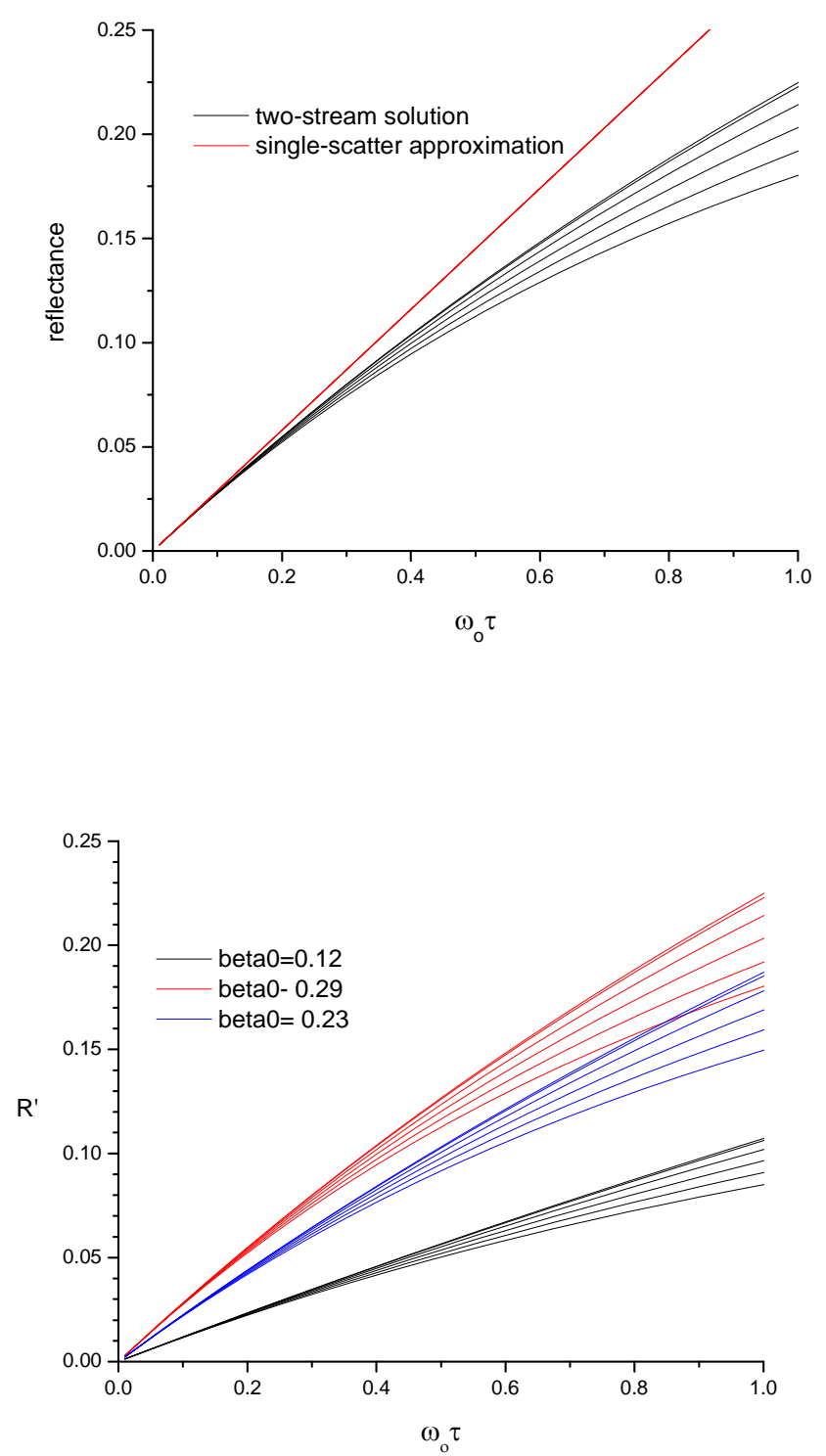
Dependence of TOA radiances on:
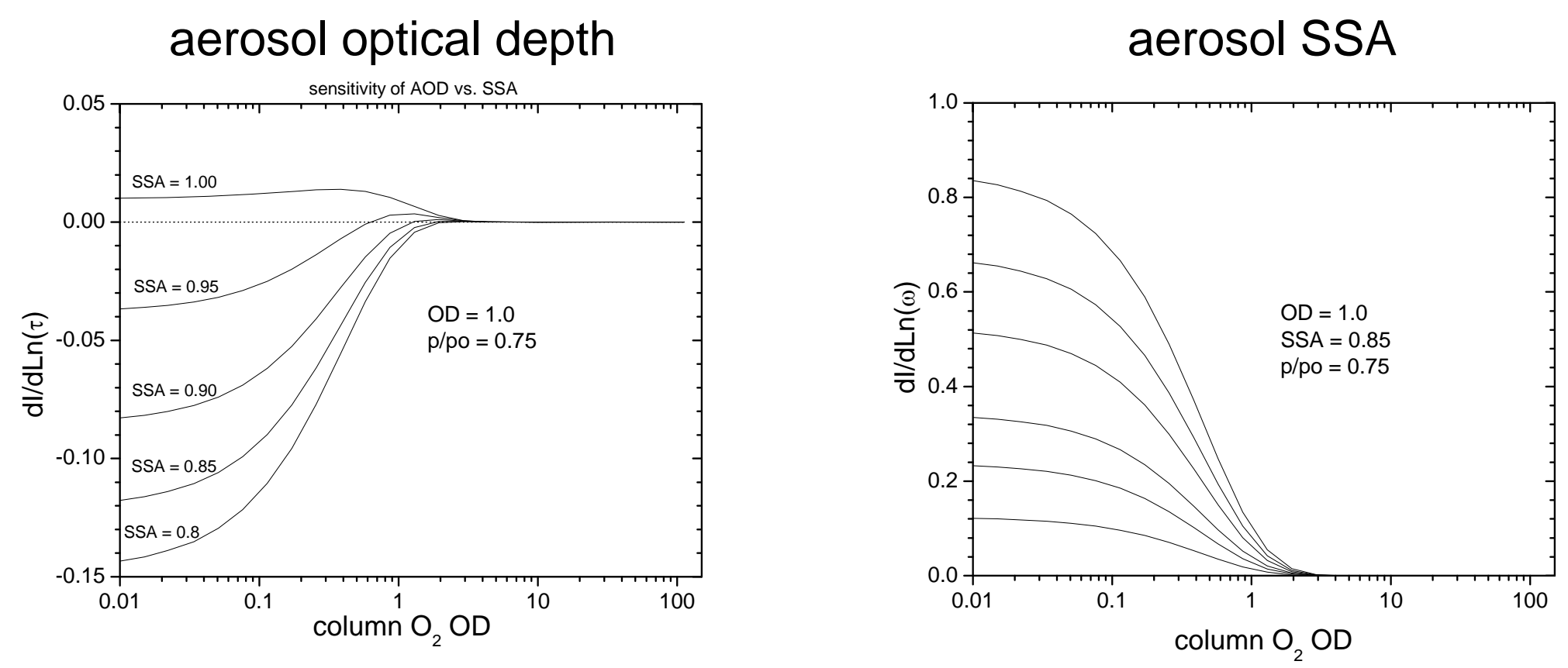


\section{Optimal Estimation Retrieval}

Initial retrieval state vector:

$$
\mathbf{x}_{\mathbf{o}}=\left(\tau_{\mathrm{a}}, \omega_{\mathrm{o}}, \alpha_{\mathrm{sfc}}\right)
$$

Forward model:

$$
\mathbf{y}=F(\mathbf{x}, \mathbf{b})+\varepsilon
$$

Atmospheric description, including lidar constraints

Iterative retrieval based on cost function:

$$
\chi^{2}=(\mathbf{F}(\mathbf{x}, \mathbf{b})-\mathbf{y})^{T} \mathbf{S}_{\epsilon}^{-1}(\mathbf{F}(\mathbf{x}, \mathbf{b})-\mathbf{y})+\left(\mathbf{x}-\mathbf{x}_{a}\right)^{T} \mathbf{S}_{a}^{-1}\left(\mathbf{x}-\mathbf{x}_{a}\right)
$$

Forward model uses a vector radiative transfer model based on the successive order of scatter method (Zhai et al., JQSRT, 2010) 


\section{In practice:}

Model for instrument SNR:

$$
\begin{gathered}
N(I)=I_{\max } \cdot \sqrt{\frac{I}{I_{\max }} \cdot C_{\text {photon }}^{2}+C_{\text {background }}^{2}} \\
\Phi=\sqrt{\sum_{i}\left(\frac{\left(I_{I}^{l}(i)-I_{I}^{m}(i)\right)^{2}}{C_{I}^{m}}\right)+\sum_{j}\left(\frac{\left.x_{j}-x_{j, a}\right)^{2}}{S_{j, a}}\right)^{2}}
\end{gathered}
$$




\section{Forward Model and Retrieval Algorithm}

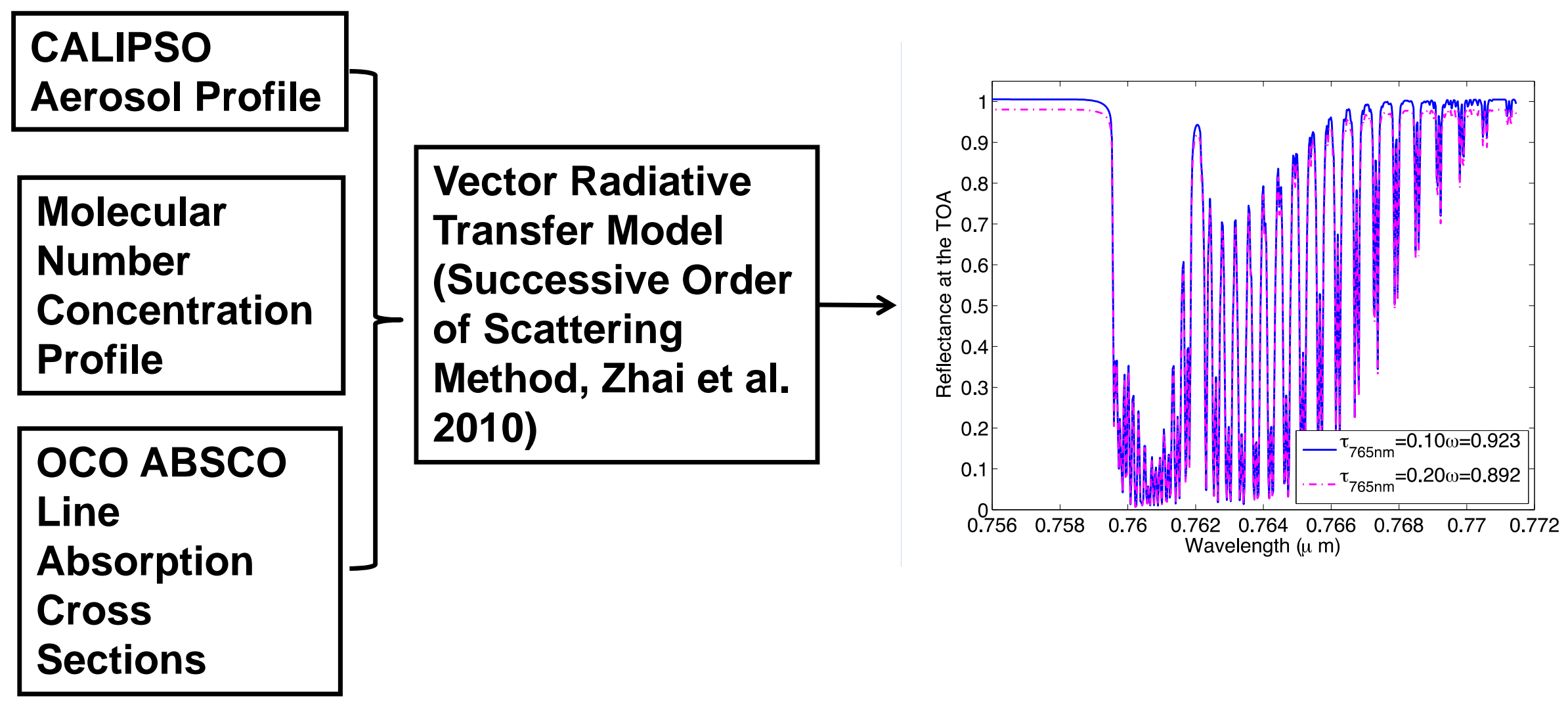

Levenberg-Marquardt Algorithm is used to minimize differences between measurements and forward model simulations. The state parameters are:

Aerosol Optical Depth

Aerosol Single Scattering Albedo

Ground reflection Albedo 
- But: even high-resolution A-band spectra have limited information content

- For an aerosol layer over a non-black surface, radiances depend on: $\tau, \mathbf{g}, \omega_{0}, \alpha_{\mathrm{sfc}}$, and aerosol vertical distribution

- Information content analysis shows 4-5 parameters at best can be derived from an OCO-like spectrometer (Heidinger and Stephens, 2000)

- Lidar provides constraints allowing improved retrievals of aerosol properties

- Scene ID (identification of layering, cloud masking)

- Aerosol vertical distribution 


\section{OCO-2 now in the A-Train: new possibilities}

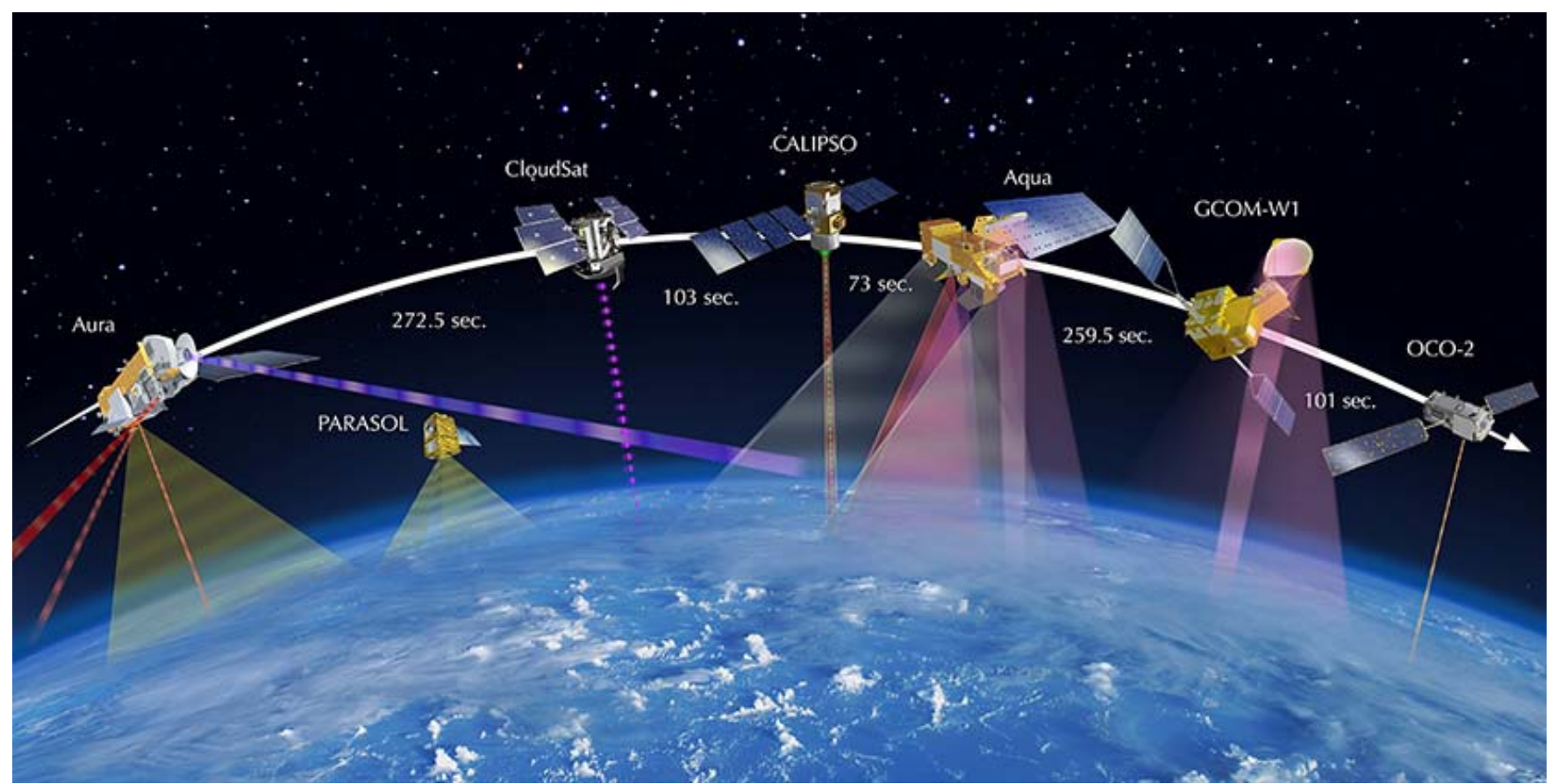

OCO-2 carries 3 spectrometers

$2.1 \mathrm{um}$ (strong $\mathrm{CO}_{2}$ )

1.6 um (weak $\mathrm{CO}_{2}$ )

0.76 um (oxygen A-band)
A-band spectrometer performance similar to that planned for CALIPSO:

$$
\begin{aligned}
& \Delta \lambda=0.044 \mathrm{~nm} \\
& \text { SNR }>200 \\
& 1-k m \text { IFOV }
\end{aligned}
$$


We have incorporated recent developments from the OCO-2 team:

- Development of improved oxygen absorption line coefficients (ABSCO look-up table)

- OCO-2 requires modeling spectra to < 1\% accuracy

- Better accounting for line mixing, collision-induced absorption, $\mathrm{O}_{2}-\mathrm{H}_{2} \mathrm{O}$ broadening, non-Voigt lineshapes

- Improved high spectral resolution solar spectrum 


\section{Retrieval from OCO-2 A-band spectra (synthetic data) assuming aerosol model is known \\ (using lidar to constrain aerosol model)}
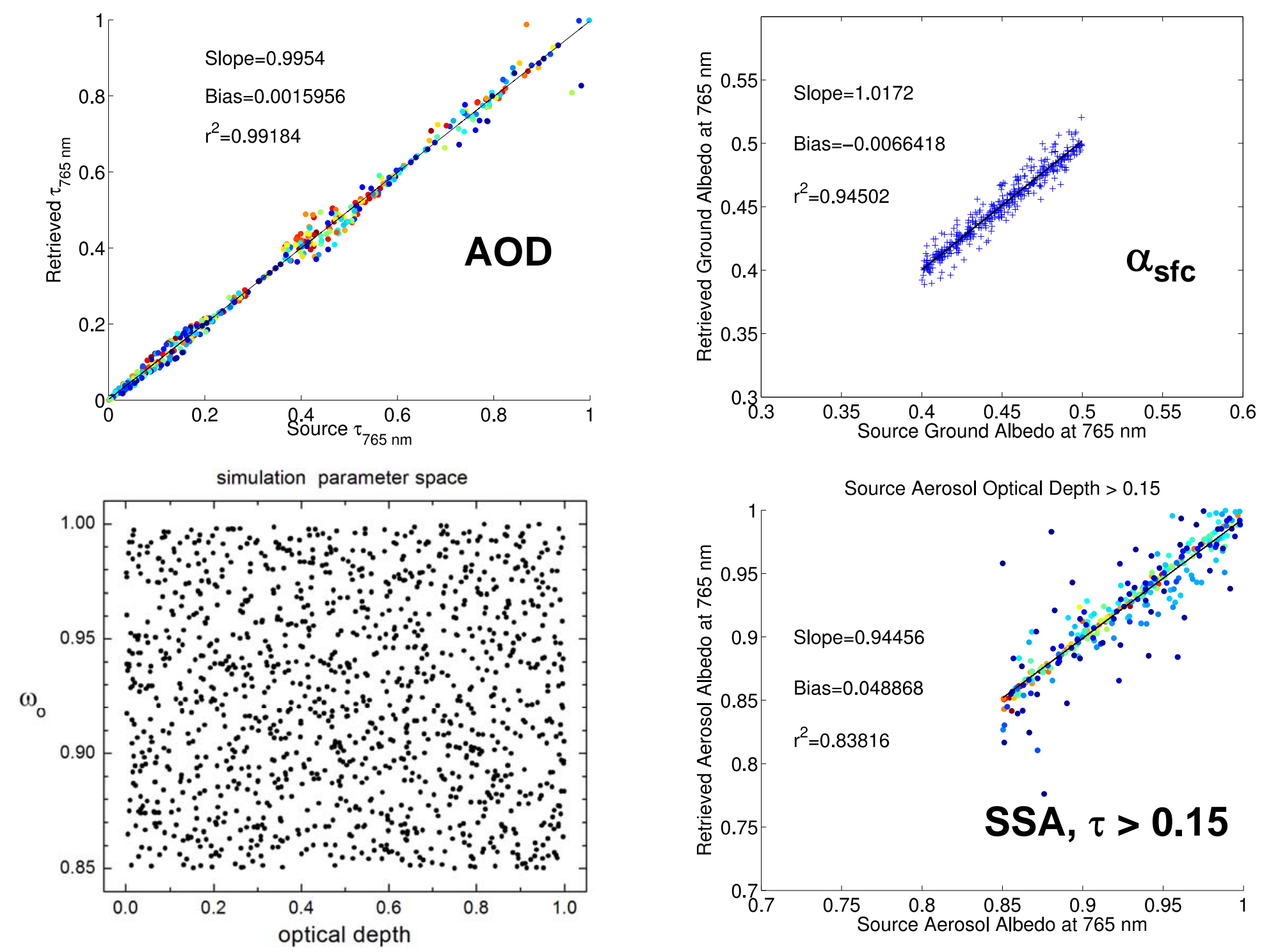


\section{Retrieval from OCO-2 A-band spectra (synthetic data) assuming aerosol model is unknown}
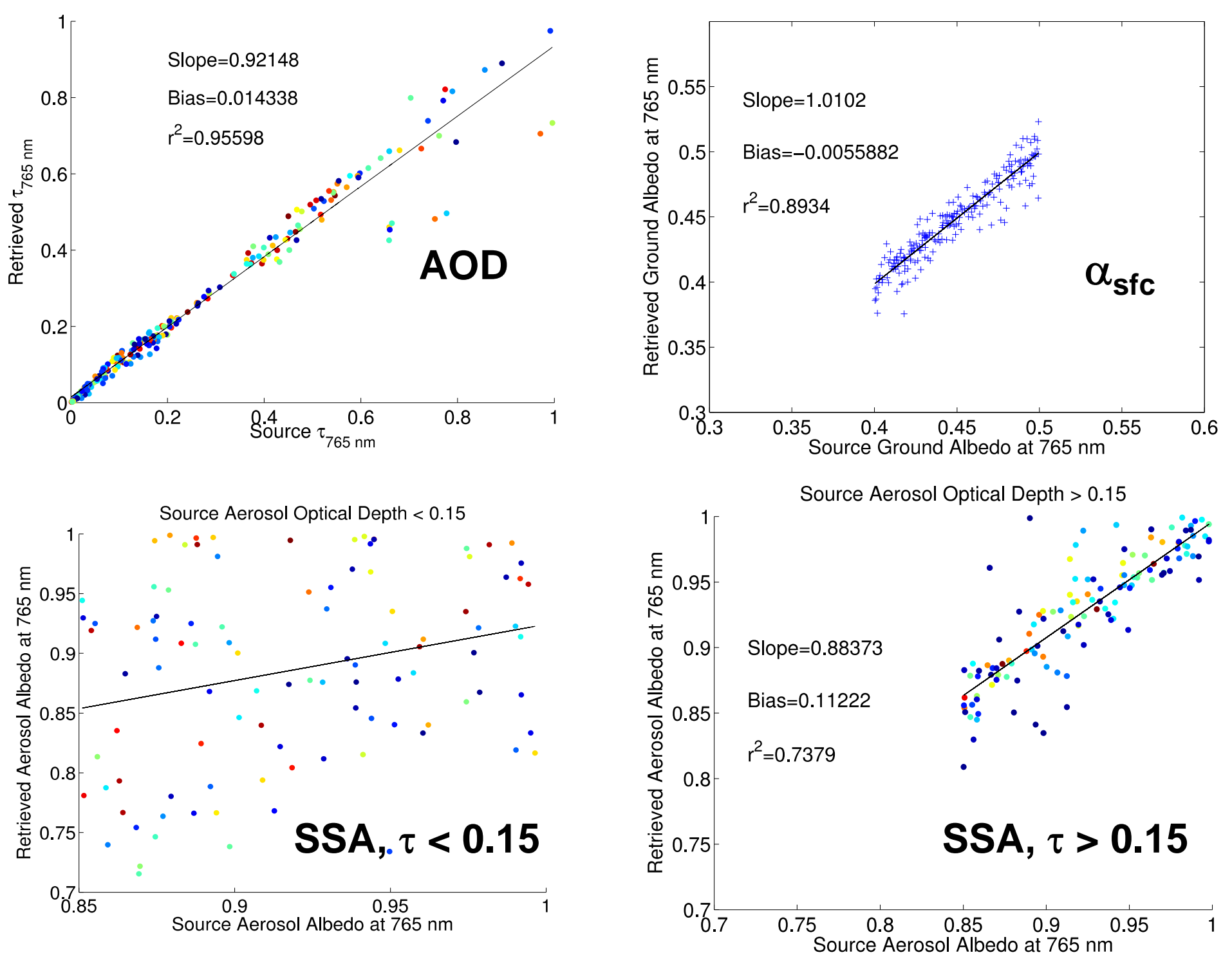


\section{Filtered simulation results}
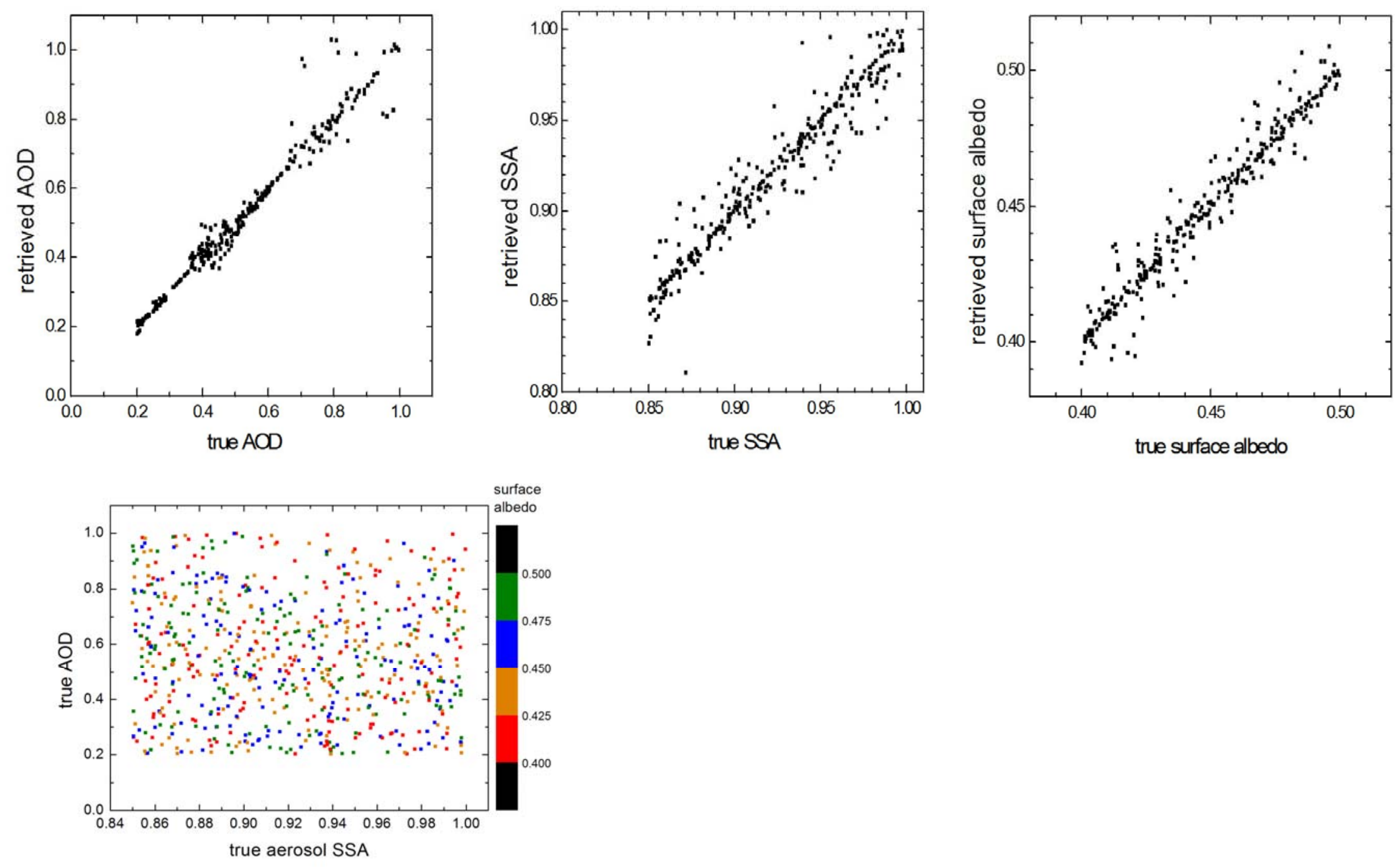


\section{Summary}

Results from 964 retrieval simulations with AOD $>0.2$, as a function of the degree of filtering applied.

$\mathrm{N}$ is the number of samples which pass the filter criteria.

\begin{tabular}{|c|cc|c|c|c|c|c|} 
Flag & \multicolumn{2}{|c|}{ AOD (\%) } & \multicolumn{2}{c|}{ SSA } & \multicolumn{2}{c|}{ Surface albedo } & \\
Value & \multicolumn{2}{|c|}{ Bias } & Std Dev & \multicolumn{2}{c|}{ Bias } & Std Dev & \multicolumn{2}{c|}{ Bias } & Std Dev & N \\
\hline 1 & 0.093 & 6.6 & -0.0016 & 0.012 & -0.00086 & 0.0069 & 300 \\
\hline 2 & -2.2 & 16.0 & -0.00013 & 0.019 & 0.0021 & 0.014 & 477 \\
\hline 4 & -1.1 & 21.2 & -0.00046 & 0.027 & 0.0014 & 0.015 & 671 \\
\hline
\end{tabular}


OCO-2 launch: 2 July 2014
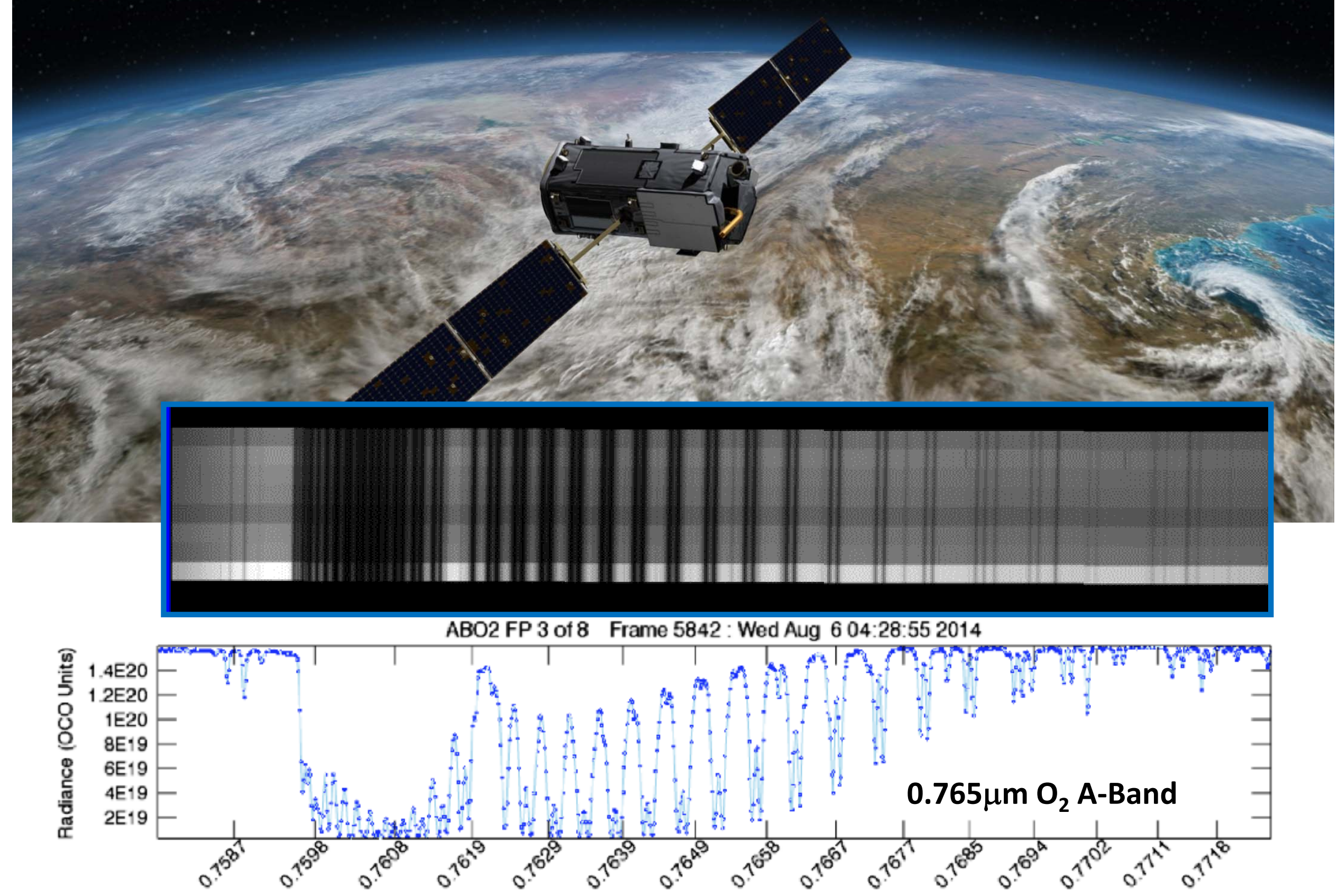


\section{Practical Considerations}

- Current status of Level 1b product indicates data quality will be sufficient

- Spectral calibration (Doppler shifts, dispersion) well in hand

- SNR currently about 400:1

- Radiometric calibration exceeds what we require

- OCO-2 team still working some details

- Bringing solar model into agreement with OCO-2 high spectral resolution observations

- Work is continuing to reduce residuals in observed high-resolution $\mathrm{O}_{2}$ spectrum 


\section{(Preliminary) Conclusions}

- Have tried to incorporate realistic instrument characteristics into retrieval simulations

- AOD retrieval performance appears to be good, even at very low OD

- The ground albedo retrieval is also very good

- Aerosol single scattering albedo retrieval performance is promising, for $\mathrm{AOD}>0.2$

- OCO-2 satellite has just moved into formation with CALIOP

- Co-located data now available 


\section{(Preliminary) Conclusions}

- Have tried to incorporate realistic instrument characteristics into retrieval simulations

- AOD retrieval performance appears to be good, even at very low OD

- The ground albedo retrieval is also very good

- Aerosol single scattering albedo retrieval performance is promising, for AOD $>0.2$

- OCO-2 satellite has just moved into formation with CALIOP

- Co-located data now available

\section{Many thanks to Dave Crisp, Dave Pollack and the OCO/OCO-2 team}

